\title{
Going "open" with Mesoscopy: a new dimension on multi-view imaging
}

\author{
Emilio Gualda • Nuno Moreno • Pavel Tomancak • \\ Gabriel G. Martins
}

Received: 9 December 2013 / Accepted: 12 December 2013 / Published online: 18 January 2014

(C) Springer-Verlag Wien 2014

\begin{abstract}
OpenSPIM and OpenSpinMicroscopy emerged as open access platforms for Light Sheet and Optical Projection Imaging, often called as optical mesoscopy techniques. Both projects can be easily reproduced using comprehensive online instructions that should foster the implementation and further development of optical imaging techniques with sample rotation control. This additional dimension in an open system offers the possibility to make multi-view microscopy easily modified and will complement the emerging commercial solutions. Furthermore, it is deeply based on other open platforms such as MicroManager and Arduino, enabling development of tailored setups for very specific biological questions. In our perspective, the open access principle of OpenSPIM and OpenSpinMicroscopy is a game-changer, helping the concepts of light sheet and optical projection tomography (OPT) to enter the mainstream of biological imaging.
\end{abstract}

Keywords Light sheet microscopy $\cdot$ Optical projection tomography $\cdot$ Fluorescence imaging $\cdot$ Microscopy .

Mesoscopy $\cdot$ Open source

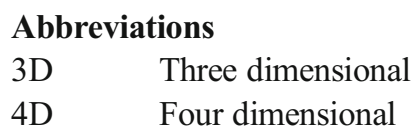

Handling Editor: J. W. Borst

E. Gualda $\cdot$ N. Moreno $(\bowtie) \cdot$ G. G. Martins

Instituto Gulbenkian de Ciência, Rua da Quinta Grande 6,

2780-156 Oeiras, Portugal

e-mail: moreno@igc.gulbenkian.pt

P. Tomancak

Max Planck Institute of Molecular Cell Biology and Genetics, Pfotenhauerstrasse 108, 01307 Dresden, Germany

G. G. Martins

Centro de Biologia Ambiental, Faculdade de Ciencias da Universidade de Lisboa, 1749-016 Campo Grande, Portugal
ASI Applied Scientific Instrumentation

CAD Computer aided design

CCD Charged coupled device

$\mathrm{CO}_{2} \quad$ Carbon dioxide

$\mathrm{CPU} \quad$ Central processing unit

CT Computed tomography

DSLM Digital scanned light microscopy

EMBL European Molecular Biology Laboratory

Gb Gigabyte

GPU Graphics processing unit

I/O Input/output

iSPIM Inverted selective plane illumination microscopy

KIT Karlsruhe Institute of Technology

LED Light-emitting diode

mSPIM Multidirectional selective plane illumination microscopy

NA Numerical aperture

OPFOS Orthogonal-plane fluorescence optical sectioning

OPT Optical projection tomography

PMT Photon multiplier tube

RAMM Rapid automated modular microscope

sCMOS Scientific complementary metal-oxide semiconductor

SIM Structured illumination microscopy

SPIM Selective plane illumination microscopy

$\mathrm{Tb} \quad$ Terabyte

\section{Introduction}

Acquiring high-resolution three-dimensional (3D) mesoscopic images is a goal for many biological research areas. The type of samples used range from cell multilayer assemblies to whole embryos, with which light penetration remains a challenge. Moreover, for live imaging reduced photodamage and fast 
acquisition rates are required. Point scanning confocal (Cremer and Cremer 1978) and two-photon (Denk et al. 1990) microscopy can be used for large specimens but excessive photodomage, caused by long exposures and poor sensitivity, diminishes or takes out completely the biological relevance of any process.

Light sheet imaging has emerged as a promising alternative that overcomes those limitations (Huisken and Stainier 2009). The uniqueness of its optical configuration, with uncoupled illumination and detection axis and combined with fast camera based detection, minimizes the amount of light entering the sample. The usage of scientific complementary metal-oxide semiconductor (sCMOS) cameras and fast piezo stages have allowed for acquiring individual multi-view datasets in a few seconds, and making it feasible to track of fast biological processes with subcellular resolution. In addition, the ability of rotate the sample in order to obtain multiview imaging, increases the quality of the $3 \mathrm{D}$ datasets and the penetration depth. Sample rotation is a characteristic shared with other mesoscopic imaging techniques such as optical or electron tomography (Messaoudil et al. 2007; Sharpe et al. 2002). Sample rotation adds an extra dimension till recently not available in any commercial and open source data acquisition software. Two different approaches, OpenSPIM and OpenSpinMicroscopy offer a platform which facilitates the implementation of light sheet and optical projection tomography (OPT) systems using open software and hardware solutions while enabling sample rotation.

\section{Light sheet imaging setups}

In a light sheet system a laser beam is shaped and focused as a thin "sheet of light", which is positioned to coincide with the focal plane of the detection objective. Therefore, fluorescence is only detected from the observed slice. As the light sheet can be tailored to the micron range, it achieves good optical sectioning and out-of-focus light suppression whereas lateral resolution is limited only by the numerical aperture (NA) of the detection objective. The use of charged coupled device (CCD) or sCMOS cameras enables higher sensitivity and speed compared to photon multiplier tube (PMT)-based detectors. Since only a fraction of the sample is illuminated during acquisition, the phototoxicity is significantly reduced compared to other fluorescence microscopy techniques.

Light sheet imaging setups have developed rapidly during the last two decades since the first designs of Voie (orthogonal-plane fluorescence optical sectioning [OPFOS]) (Voie et al. 1993) and Ernst Steltzer (oblique illumination confocal microscope or confocal theta microscope) (Lindek et al. 1994). The current interest in the technique derived mostly from the implementation of Huisken et al. (2004), from which the broadly used acronym SPIM (selective plane illumination microscopy) was derived. The authors combined the idea of light sheet illumination and detection with sample rotation to obtain multiple 3D stacks (views) of whole living embryos over time. Application of a multi-view configuration, the axial resolution is no longer limited by the detection objective, making it a prominent alternative to conventional 3D approaches such as confocal or multiphoton microscopy.

More recently, light sheet imaging setups can also pertain to a laser scanning module instead of an instantaneous sheet of light generated by a cylindrical lens. The idea behind this configuration, known as digital scanned light microscopy (DSLM) (Keller et al. 2008), is to generate a quasi-plane of light using a laser scanner that rapidly moves a micrometer beam vertically through the specimen. The entire power of the light source is focused onto a single line and therefore enabling the use of lasers with one order of magnitude less power. Also, as it does not rely on apertures to form the laser profile, it reduces optical aberrations, increases image quality and consequently leads to better quantification of the fluorescence signal. DSLM allows the generation of intensity-modulated patterns, which can be used to enhance contrast in highly scattering specimens (Breuninger et al. 2007; Keller et al. 2010). Furthermore, it can be combined with two-photon excitation (Keller et al. 2010; Truong et al. 2011) or narrow self-reconstructing Bessel beams for illumination (Fahrbach et al. 2013a; Gao et al. 2012; Olarte et al. 2012; Planchon et al. 2011). To deal with shadowing effect on the side of the sample furthest from the illumination, other illumination configurations have been proposed. In mSPIM (Huisken and Stainier 2007) or Ultramicroscope (Dodt et al. 2007), the scattering attenuation is compensated by illuminating from opposing directions, but still perpendicular to the detection objective, enabling a better illumination distribution over sample thickness.

Light sheet - exploiting it to the limits

Recent advance in instrumentation development have led to advanced light sheet systems aiming to achieve faster acquisition, increased penetration, resolution and signal to noise ratio. This has been achieved by clever small adaptations to standard setups or by combining with other common techniques. For example, introducing a slit in the detection path (Silvestri et al. 2012) the advantages of light sheet illumination and confocal detection are combined. This approach can be mimicked using a rolling shutter of a sCMOS camera (Baumgart and Kubitscheck 2012). Another way to increase image contrast is background rejection with structured illumination microscopy (SIM) (Breuninger et al. 2007; Keller et al. 2010) or HiLo microscopy (Mertz and Kim 2010).

While thinner sheets lead to better axial resolution, they are also accompanied by a more pronounced non-uniform light sheet thickness across the field of view. An alternative is the usage of self-reconstructing beams or Bessel beams (Gao et al. 2012; Olarte et al. 2012; Planchon et al. 2011), stimulated 
emission depletion (Friedrich et al. 2011) or 3D interfering structured (Lei and Zumbusch 2010) light sheet microscopy. However, it does not improve lateral resolution, for that purpose molecular localization microscopy has already been applied to light sheet imaging (Zanacchi et al. 2011).

Fast data acquisition is also an important aspect that has been addressed using light sheet imaging. Introducing a piezodriven objective greatly reduces the settle time for each plane acquisition step. Light sheet position can be either controlled with a galvo mirror (Ahrens et al. 2013; Keller et al. 2008) or attaching the planar illumination optics directly to the detection objective (Holekamp et al. 2008)). Alternatively, and without moving the objective lens, the light sheet can be scanned through the sample using a galvo mirror and synchronized with tunable lens (Fahrbach et al. 2013b). One of the latest developments is omnidirectional microscopy (Weber and Huisken 2012), i.e., the use of a fixed set of four lenses (two for illumination and two for detection) and two cameras in order to minimize the time-consuming specimen rotation (Krzic et al. 2012; Tomer et al. 2012). Other fast acquisition implementations for light sheet imaging has allowed obtaining new relevant quantitative information such as diffusion and interactions using single molecule tracking (Ritter et al. 2010) or fluorescence correlation spectroscopy (Wohland et al. 2010).

\section{Optical projection tomography}

Optical tomography micro/mesoscopy (most often referred to as optical projection tomography) operates in a fundamentally different manner than light sheet or point-scanning confocal microscopes, which collect images of individual slices sequentially, yet both approaches share sample rotation. Applications of OPT involves simultaneous illumination and detection, in the same way as a sample is imaged in a conventional widefield fluorescence microscope/macroscope. Several images of the full "projection" of the sample are captured from multiple angles, and virtual sections — or slices — are reconstructed computationally a posteriori through a process known as back-projection reconstruction (Sharpe et al. 2002). In order to identify anatomical details inside the sample, they must be visibly superimposed on the projection, so typically the technique relies on the observation of highly-transparent samples embedded in a medium with the same refractive index as glass. Any aberration or light dispersion, which is inevitable in live (aqueous) samples - even in transparent embryos such as zebrafish - results in artifacts during slice reconstruction. Therefore, OPT is feasible mostly with chemically fixed and tissue-cleared samples. Attempts to image live samples with OPT provided images which are no better than the ones obtained with a confocal microscope. Despite of this limitation, optical tomography can provide valuable information in vivo at the macroscopic level; examples of such applications are techniques generally referred to as optical diffuse tomography (Ntziachristos 2010), such as bioluminescence tomography (Gu et al. 2004; Wang et al. 2004) or fluorescence molecular tomography (Ntziachristos 2010). So far, these techniques have found applications mostly in pre-clinical research as they provide primarily functional information with very limited anatomical detail in the meso- to microscopic scale.

Optical tomography seems to be particularly useful for mesoscopic-level samples (1-10 mm thickness) such as whole vertebrate embryos or small animal organs. The apparatus is similar to a conventional microscope with the exception that in this case the stage allows rotation sample rotation. For this reason, new setups or adaptation from an existing micro/ macro/stereoscope are relatively easy to implement.

\section{Platforms and components}

\section{Commercial systems}

There are currently three available commercial light sheet systems: LaVision BioTec's Ultramicroscope, based on the Dodt prototype (Dodt et al. 2007). It consists of a dual-sided light-sheet illumination system with cylindrical lenses mounted on a stereoscope with upright detection path. It provides large working distances up to $5.5 \mathrm{~mm}$ and variable magnification from $1.26 \times$ to $12.6 \times$. It has been mainly used for imaging large fixed and cleared specimens (Hagerling et al. 2013; Jahrling et al. 2009), but it is in principle also applicable for live imaging.

In 2012, the microscopy company Zeiss released the Lightsheet Z1, a light sheet system with features similar to the mSPIM setup (Huisken and Stainier 2007). This microscope is the only commercial system that allows multi-view imaging through sample rotation. Illumination is performed sequentially from both sides with a combination of cylindrical optics and a beam scanning mechanism to reduce stripes on the image due to sample absorption (Huisken and Stainier 2007). The detection axis is equipped with zoom optics allowing fine adjustments of magnification and field of view to wide range of samples. It also provides specialized software to improve resolution of the processed dataset by recombining information from different views using fluorescent beads as fiduciary markers (Preibisch et al. 2010) and the use of deconvolution tools to further improve the image quality. This system is presented on a standalone sealed box allowing incubation with temperature and $\mathrm{CO}_{2}$ control, and includes different detection (magnifications of $5 \times, 20 \times, 40 \times, 63 \times$ ) and illumination $(5 \times, 10 \times)$ objective lenses.

Applied Scientific Instrumentation (ASI) released the rapid automated modular microscope (RAMM) system based on the inverted selective plane illumination microscopy (iSPIM) (Wu et al. 2011). This modular option can be coupled to any 
existing inverted microscope by replacing the transmitted illumination tower with a new pillar to steer the sheet onto the sample and obtain $\mathrm{Z}$ sections. All other components such as camera, microscope and light sheet generation are not provided by ASI.

Although X-ray based micro-tomography has been available commercially for several years, however an optical version was only developed in 2002 (Sharpe et al. 2002). A commercial version was then offered by Bioptonics, through a partnership with SkyScan, a manufacturer of microCT X-ray scanners. Since 2012, this system is no longer commercially available, and it is only possible to request services to scan samples.

\section{Custom and open systems}

Establishing a dedicated system to a single application for light sheet or OPT is extremely easy compared to other imaging techniques. The arrangement for broader applications on a single machine can lead to engineering complexity that might not compensate the investment on the development. However, the delay in commercial realisation of these technologies prompted the widespread implementation of custom made systems. The basic building blocks of light sheet and OPT microscopes are: laser or LED illumination, objectives and lenses, camera, a system to create the light sheet(cylindrical lens or galvo mirror)(Greger et al. 2007) and a sample holder for allowing sample positioning including rotation. They may also include acousto-optic systems to control light dosage and piezo stages for the sample or objective positioning.

Although many custom-built systems are described in detail in many publications (Keller et al. 2010; Tomer et al. 2012) replicating a functional set-up based on these instructions may be challenging especially for researchers lacking experience in optical technology development. One way to minimize the problem of implementation and control of those systems is to adopt an open source approach. Open source software for image analysis, like ImageJ/Fiji (Schindelin et al. 2012) or to image acquisition and microscope control, such as Micromanager (Edelstein et al. 2010) has been the choice for many facilities to standardise image acquisition and processing. Micromanager is compatible with most of the hardware used in microscopy (cameras, shutter, laser control, etc.). However, until recently, the introduction of some essential features in Micromanager such as sample rotation (and galvo control for DSLM) and metadata handling has made this system very attractive for light sheet or OPT experiments. These features have also been in the OpenSPINMicroscopy (Gualda et al. 2013) and OpenSPIM (Pitrone et al. 2013) initiatives. Both projects provide through their webpages not only part lists, sample mounting methods and sample chamber designs, but also software to fully control data acquisition (for more details, see Figs. 1 and 2).
The innovative approach of this setup consists on the use of open source hardware, i.e., Arduino microcontrollers, opening this technology to any laboratory with a minimum technical background. Arduino is a popular, open-source hardware prototyping board with an ATmega328 microcontroller, compatible with Micromanager. This low-cost (less than \$20) programmable digital $\mathrm{I} / \mathrm{O}$ board was primarily designed to extend the use of electronics to areas beyond the ones used to work with this kind of technologies. The system uses three Arduino boards with modified firmware to control a shutter, a galvo for DSLM and three stepper motors. One for sample rotation and others for filter wheels excitation and detection. This approach reduces significantly the costs of such kind of system and can be modularly upgraded, helping to the widespread of this technology for basic applications in an easy way.

Two different setups (one for SPIM/DSLM and OPT and another fully dedicated to OPT) were built using the OpenSPINmicroscopy platform. Through this web page (http://uic.igc.gulbenkian.pt/micro-dslm.htm), full access to hardware (sketches, a parts list and circuit designs) and software (plug-in code, Micromanager device adaptors and Arduino firmware) is provided. Special attention has been paid to the design of sample chambers and sample mounting, providing different designs suited for air or water dipping objective lenses. Since the samples are usually embedded in agarose in order to keep the humidity conditions, they are submerged into a waterlike environment.

This integrated platform allows the implementation of a vast array of imaging approaches and offers the versatility to image samples ranging from small organisms to whole embryos $(\sim 1-2 \mathrm{~mm})$ and larger samples up to $1-2 \mathrm{~cm}$. Also, because it uses micromanager as platform, it is able to deal with all sorts of its supported hardware.

The OpenSPIM implements a single sided illumination and single sided detection light sheet setup that fits onto an optical breadboard of $30 \times 45 \mathrm{~cm}$ (Fig. 2a), a rack (Fig. 2b) or even inside a cabin-sized suitcase (Fig. 2c). The illumination and detection arms meet at 90 degrees angle in the water-filled sample chamber (Fig. 2d). Emission filters can be easily inserted into a slit in the spacer of the detection axis (Fig. 2e). The pre-assembled 4D positioning system (Picard Industries, Albion, NY, USA) controls rotation and translation of the sample through the light sheet within the chamber (Fig. 2f). The sample is mounted in agarose and extruded from a capillary fixed in position by a cut plastic syringe serving as sample holder (Fig. 2g,h). An experienced researcher can assemble the OpenSPIM set-up in about $15 \mathrm{~min}$ (Fig. 2i).

The OpenSPIM is controlled via the popular Open Source microscopy control software Micromanager embedded in ImageJ's distribution Fiji (Schindelin et al. 2012) 
Fig. 1 a Volume reconstruction of a Bufus bufus tadpole (1 week), obtained with optical tomography by detection of green autofluorescence. Magnification scale bar represents $1 \mathrm{~mm}$. b Volume reconstruction of a flower of Ditassa burchelli obtained with optical tomography by detection of green autofluorescence.

Magnification scale bar represents $1 \mathrm{~mm}$. c Maximum intensity projection of Drosophila melanogaster embryos (transgenic line: $\mathrm{w} \mathrm{P}(\mathrm{w}+$ asl:YFP);G147 His:RFP/TM3) expressing YFP-tagged asterless (green) and RFP-tagged histones (red). Detection objective, 16×, NA 0.8. Magnification scale bar represents $100 \mu \mathrm{m}$. d Maximum intensity projection of autofluorescence from a wild type Arabidopsis thaliana. Different features such as stomata (red), leaf chloroplast (green) and (blue). The excitation wavelengths were $488,567,648 \mathrm{~nm}$ and detection filters $580 / 25 \mathrm{~m}-2 \mathrm{p}$, HQ 620/ $90 \mathrm{~m}-2 \mathrm{p}$ and ET700/50 m-2p, respectively. Magnification scale bar represents $100 \mu \mathrm{m}$. e Volume reconstruction obtained from the fusion of eight views of a fliEGFP transgenic fish showing green fluorescence in the vascular system and human tumor cells labeled with the lipophilic cell tracer carbocyanine red dye, Dil. Detection objective, $4 \times$ NA 0.13 . Magnification scale bar represents $1 \mathrm{~mm}$. f Overview of the OpenSPINmicroscopy micromanager plug-in
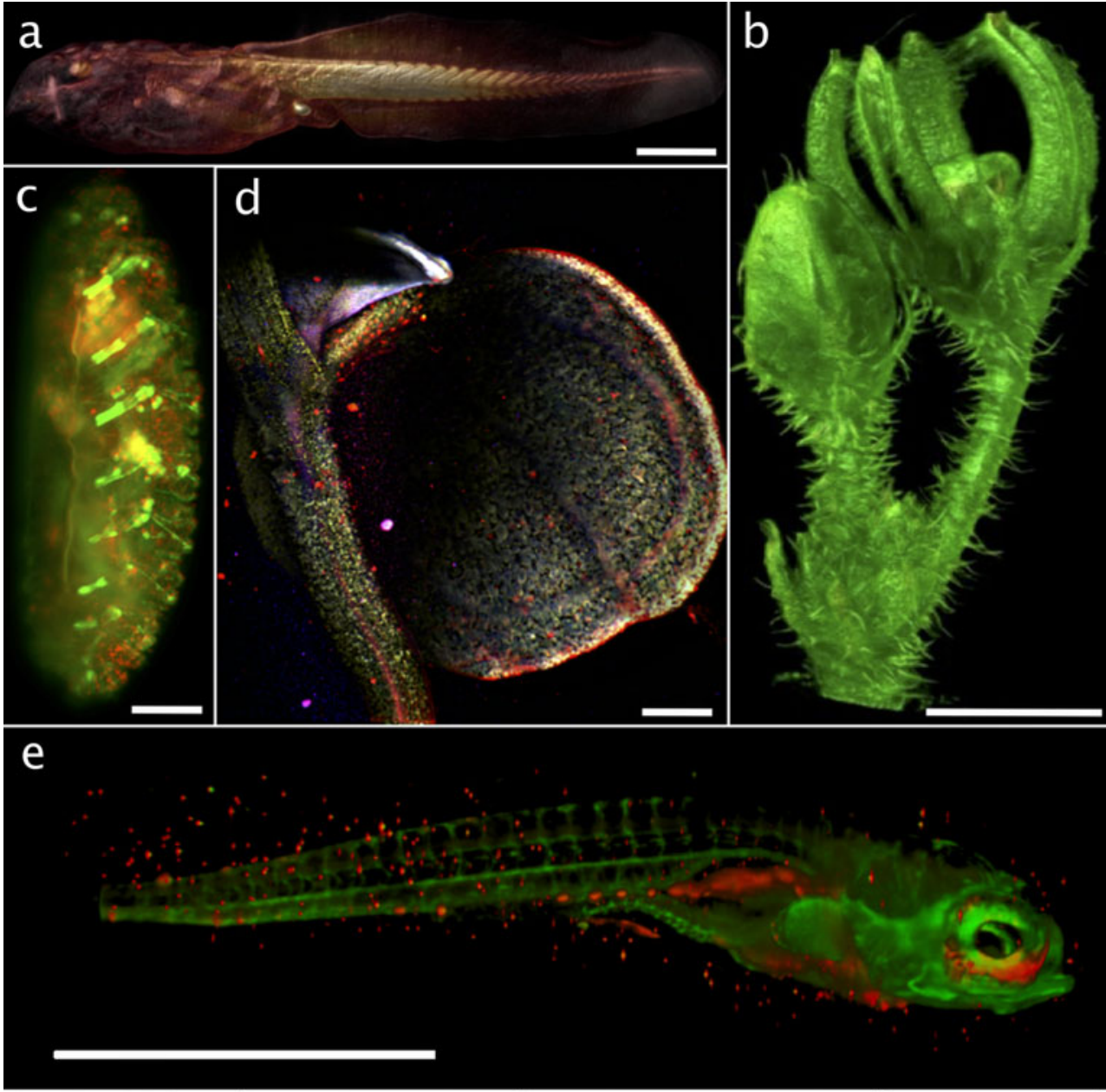

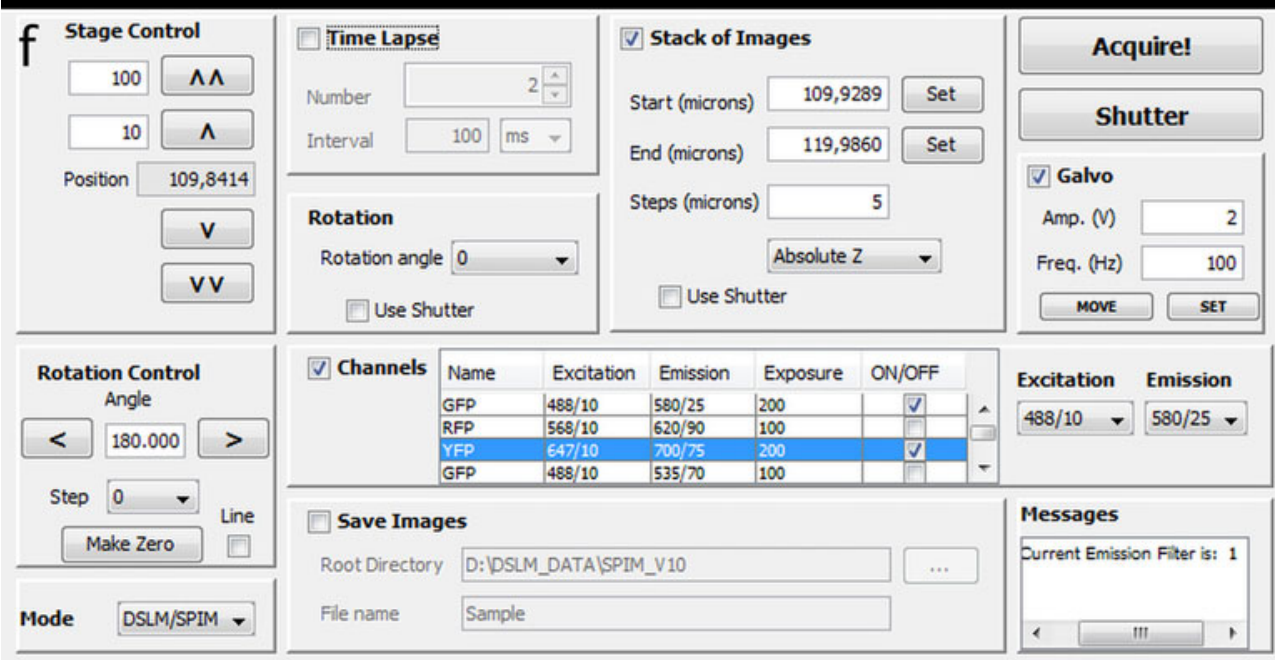

where the acquired data are available for bead based multiview reconstruction, mulitiview fusion and deconvolution (Preibisch et al. 2010; http://arxiv.org/abs/1308.0730) (Fig. 2j). OpenSPIM uses Fiji's update mechanism to distribute and update the OpenSPIM software components, which maximizes flexibility. A single laptop computer with no additional electronics is able to steer the OpenSPIM microscope adding simplicity for users without engineering background.

Together with Fiji, the OpenSPIM forms an accessible and highly adaptable open access platform for multi-view light sheet imaging in developmental, cell and evolutionary biology (Fig. 2k-n). It is meant to be a starting point for prototyping more advanced light sheet paradigms. It is an excellent 
teaching tool at all educational levels and it can provide an entry level advanced microscopy system in less developed parts of the world. OpenSPIM can be parallelised to increase the throughput of long-term time-lapse acquisitions or it can be deployed for many days to image slow development of emerging model organisms. The possibilities are limitless and only time will tell if an interdisciplinary community of researchers interested in application of light sheet microcopy will form around the OpenSPIM/Fiji hardware and software ecosystem.

\section{Dealing with terabytes}

Light sheet datasets generally do not require preprocessing routines such as denoising, deconvolution or unmixing due to their high quality. With OPT, pre-processing of image datasets is critical as any signal noise, uneven illumination or pixel saturation will result in severe artifacts during the backprojection reconstruction. Notwithstanding, the complexity of information from the anatomical embryos in toto, either live or fixed,calls for new tools to deal with data segmentation, visualisation, navigation or dynamic representation. Both light sheet and OPT can benefit tremendously from a posteriori processing and analysis such as "multi-view" registration and deconvolution (http://arxiv.org/abs/1308.0730). It requires combining the collected images into a new image dataset, which should ideally offer increased resolution in Light Sheet and sample coverage in OPT.

The first algorithms for multi-view registration were developed for tilted-view microscopy (Shaw et al. 1989) and has been adapted to light sheet microscopy in several works using one (Swoger et al. 2007) or two (Krzic et al. 2012) cameras in parallel. An open source plug-in, SPIM Registration (Preibisch et al. 2010), is also available through Fiji software package. This software, instead of sample features, uses fluorescent beads in rigid mounting medium as reference markers, enabling efficient, sample-independent registration of multiview SPIM acquisitions. The approach is used both by OpenSPIM and Zeiss Lightsheet Z1 platforms. Fiji offers also several strategies to combine the registered data into a single output image using content-based fusion (Preibisch et al. 2008) and Bayesian-based multi-view deconvolution (http:// arxiv.org/abs/1308.0730) exploiting the computing power of the graphics cards (GPU). Other fusion approaches exploit operations in Fourier space (Rubio-Guivernau et al. 2012; Swoger et al. 2007) however their availability is limited. Moreover recent designs such as the SiMView microscope (Tomer et al. 2012) also offer tailored software running in Matlab to perform four-view fusion of time-lapse or singlerun data sets recorded with bi-directional illumination and two cameras.
Fig. 2 a 3D rendering of an OpenSPIM set-up implementing single sided illumination and detection (L-OpenSPIM). b 3D rendering of a farm of four T-OpenSPIMs implementing dual sided illumination and single sided detection (concept design). c A photograph of a "SPIM in a suitcase" OpenSPIM variant. d Sample chamber consisting of Olympus $\times 10 / 0.3$ illumination and $\times 20 / 0.5$ detection objectives arranged perpendicularly in a custom made sample chamber. The chamber is filled with water and the light sheet is on. e Detection axis spacer with custom emission filter insert sticking out. f 4D USB sample positioning system with sample holder arm and pulley system translating the movement of the fourth motor into rotation. g,h Modified plastic syringe serving as a simple holder for glass capillary with specimen in agarose. i Nine steps of OpenSPIM assembly process in top-down view. $\mathbf{j}$ Schematic representation of the OpenSPIM steering software architecture. Data from the OpenSPIM microscope are collected by the OpenSPIM plug-in that extends microManager running in Fiji where the data are available for reconstruction and analysis. The design enables active feedback of onthe-fly image processing results on image acquisition on multiple levels (bidirectional arrows). The software is loaded from a dedicated update site. k 3D rendering of OpenSPIM recording of Drosophila embryogenesis. Drosophila embryos, expressing His-YFP in all cells, imaged from five angles every $6 \mathrm{~min}$ from gastrulation until embryo movement prevents further meaningful imaging. I Example of unprocessed raw OpenSPIM data, a single slice through blastoderm stage Drosophila embryo expressing His-YFP. m 3D rendering of sea star larva stained with anti-acetylated tubulin antibody

One of the major problems with light sheet techniques is the huge amount of data acquired that must be afterwards stored and processed in order to extract relevant information. In a typical recording of developmental processes such as embryogenesis thousands of time points are needed, each consisting of hundreds of sample sections and with multiview functionality the amount of data is multiplied by factor of 4 to 8 . Moreover, new sCMOS cameras provide up to 4-5 megapixel resolution, leading to several terabytes of information per experiment. The tasks needed to extract quantitative information about cell morphology and cell behavior from the enormous datasets recorded with light sheet techniques demands automated approaches for computational image analysis (3D cell shape segmentation, cell tracking, quantification of gene expression levels, subcellular localization of proteins) in order to extract meaningful biological information. OPT datasets can also present a challenge. With the same camera, yields nearly $13 \mathrm{~Gb}$ of raw data, which then needs to be processed and a new $3 \mathrm{D}$ dataset of yet a few more $\mathrm{Gb}$ is distilled.

One of the first attempts to obtain quantitative imaging using light sheet microscopy techniques is the so called "digital embryo" developed by Stelzer's laboratory. It consisted in a set of software modules that automatically detects nuclei in the raw images by using large-scale compute cluster (at the European Molecular Biology Laboratory, EMBL and Karlsruhe Institute of Technology [KIT]), providing a first attempt of a comprehensive databases of cell positions, divisions, and migratory tracks (Keller et al. 2008). Through this method, relevant information from raw data representing several 
a

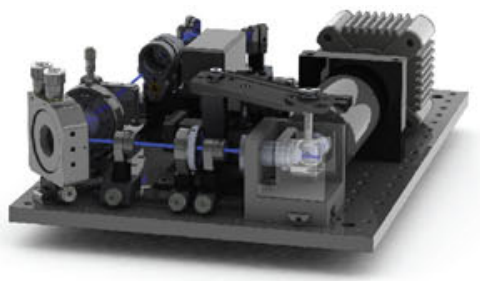

C
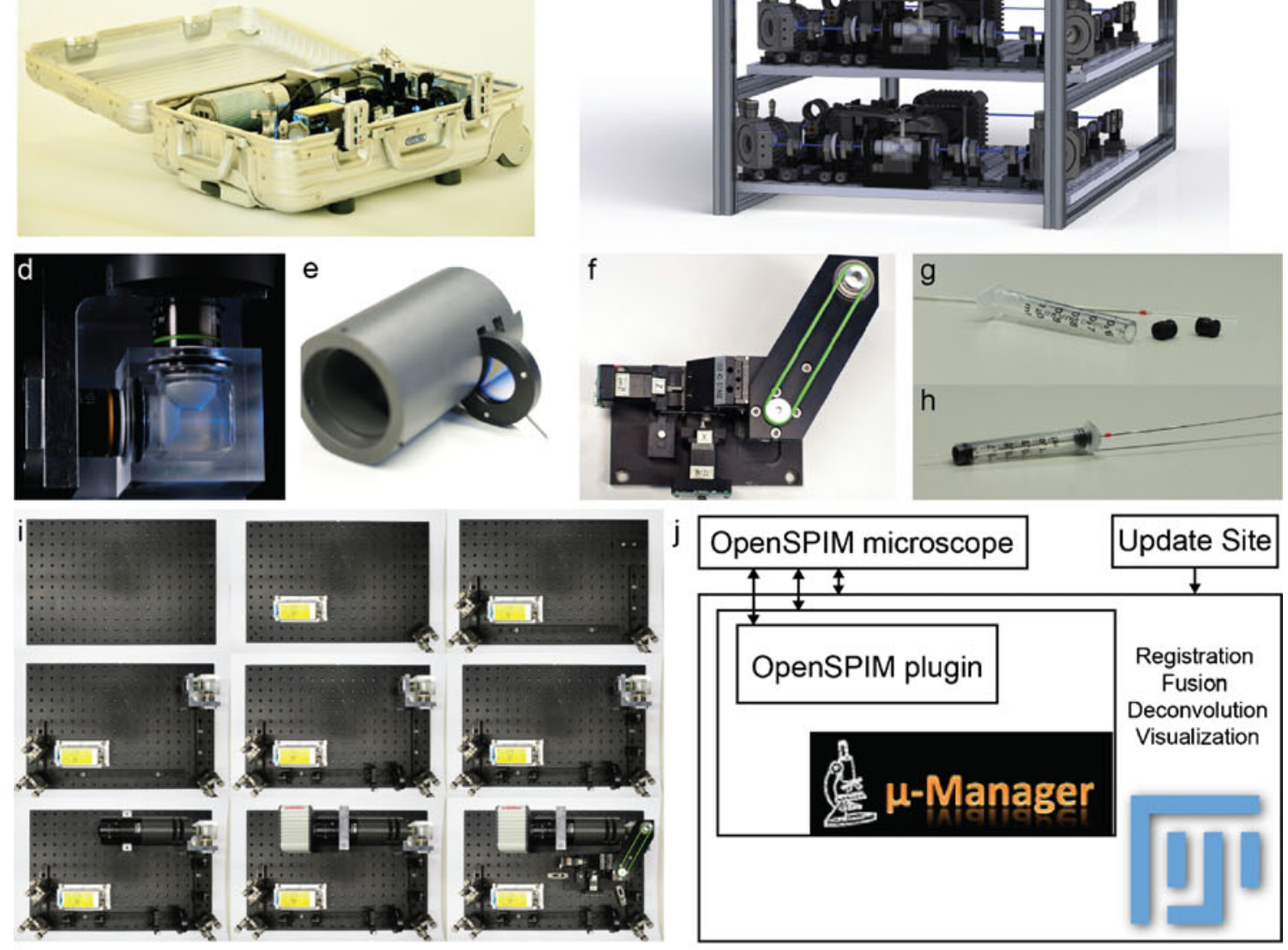

$\mathrm{k}$ gastrulation germ band extension germ band retraction dorsal closure head involution
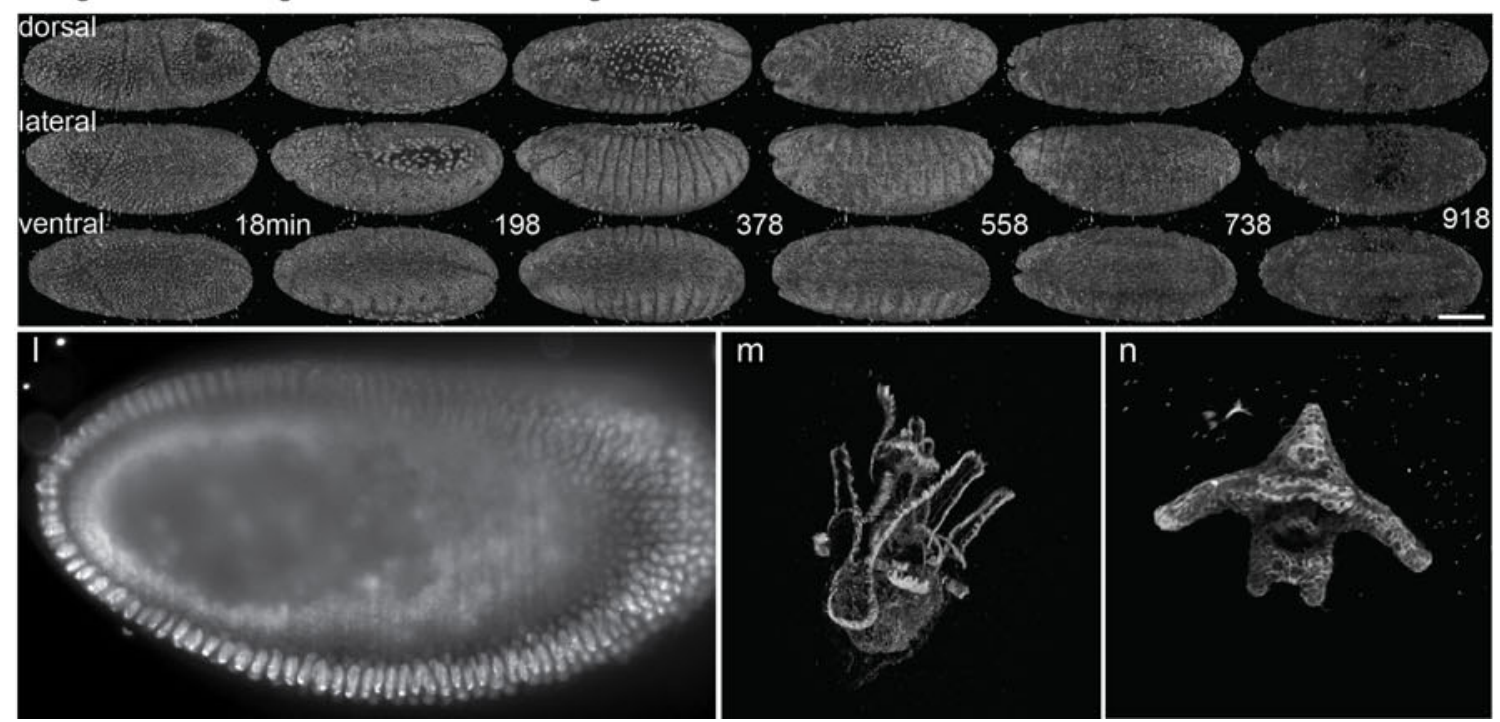
terabytes can be extracted and potentially reduced to only hundreds of megabytes. However, in order to be able to safely delete the raw data the algorithms for segmentation and tracking have to be perfect. This remains an open challenge for the bioimage analysis community. Nevertheless those databases are public and have been used by other groups to evaluate new metrics to measure the development rate of zebrafish embryos (Hoh et al. 2013).

For some specific samples, such as zebrafish and Drosophila embryos, where most cells are confined to the surface of the early stages embryo, one interesting alternative to display datasets are two-dimensional projections of the data of interest. Drosophila embryo surface was "unrolled" obtaining a projection of the epidermal layer (Krzic et al. 2012). For zebrafish (Schmid et al. 2013), the endodermal cells form a single cell layer allowing approximating its shape by a sphere. Radial maximum intensity projections are computed in real time during acquisition without saving any raw image data, reducing the amount of data from $\mathrm{Tb}$ to $\mathrm{Gb}$. This approach facilitates the analysis of cell division, migration and differentiation. Different cartographic projections are possible depending on the experiment, revealing characteristic migration patterns of the early endoderm.

For OPT, back-projection reconstruction can be performed using the same algorithms used for the medical X-ray images, and there are multiple solutions available not only commercial but also open-source. Before back-projection, the dataset containing the multi-views needs to be aligned so that the axis of rotation is perfectly vertical and centered within the field of view. Moreover the images have to be processed for flat-field correction and noise reduction (e.g., by filtering hot and/or outlier pixels). For the back-projection reconstruction of sections, SkyScan offers a non open-source software called NRecon, which is optimized for reconstruction with GPUs, making it $>10 \times$ faster than the reconstruction done with $\mathrm{CPU}$ processing a major advantage for processing numerous datasets or optimizing reconstruction parameters. An easy to use open source solution exists in the form of a plug-in for the popular ImageJ/FIJI software, known as Radon transform, which in our experience produces superb reconstructions of optical projection datasets. The processing is done exclusively in the CPU, so typically it will require several hours or days to process a full dataset, even with current high end CPUs. One other possibility freely available and integrated with ImageJ/ FIJI is the TomoJ package (Messaoudil et al. 2007), which, besides the classical filtered back-projection reconstruction, can also reconstruct projection datasets with iterative algorithms.

Large specimen often comprises different levels of contrast in different tissues. A consequent common problem is to properly resolve highly contrasting structures because more faint details cannot be properly captured (Fei et al. 2012) A proposed method is the use of laser instead of diffuse incident light, enabling multiple images of each multi-view angle to be generated with higher dynamic range and higher photon efficiency (Lorbeer et al. 2011). Many artifacts in OPT are caused by specimen movement, misalignments and positioning of the sample in the center of the field of view. These have been addressed by (Birk et al. 2011; Birk et al. 2010; Cheddad et al. 2012; Vinegoni et al. 2009; Walls et al. 2007).

Both techniques - light sheet imaging and OPT — render output data that can be used far beyond the needs of most articles. Not surprisingly, much of the work done so far with these techniques has been to revisit problems of morphogenetic processes or morphological characterisation. The superb detail of the 3D datasets and the needs to share it beyond the constraints of regular scientific manuscripts has led to new avenues for sharing anatomical data generated from these techniques. Examples of such projects are the well known "Mouse Atlas Project" and "AnatomyPortal.net/fishnet".

\section{Conclusions}

Due to the low photodamage, fast acquisition rates and large fields of view with subcellular resolution, light sheet imaging allows the study of in vivo processes and their relationship with structural features in fluorescently labeled living specimens. OPT is easier and more inexpensive to implement, and is especially useful for larger samples (few $\mathrm{mm}$ to $\mathrm{cm}$ ) providing superb 3D datasets with isotropic resolution. Both methods have been successfully used in embryogenesis studies of model organisms such as zebrafish and Drosophila melanogaster and to organogenesis (Swoger et al. 2011) and cell dynamics investigations like cell migration (Schmid et al. 2013), cardiac development (Fahrbach et al. 2013b), blood flow (Wohland et al. 2010), vascular development (Jahrling et al. 2009), neuro-development or calcium imaging (Ahrens et al. 2013; Panier et al. 2013) in embryos and small organisms. Optical micro-tomography, on the other hand, seems to be better suited for analysis of anatomical detail of large specimens (typically on the mesoscopic range, where the effects of light scattering dominate the final contrast of images of in vivo samples) and has proved to be both easy and reliable to implement. Processing and analysis of optical tomography images is also relatively straightforward, given the extensive existing knowledge on processing and analysis of X-ray biomedical CT images.

As they grow in number of components, light sheet and OPT systems are becoming more increasingly complex. As commercial systems cannot by definition be kept up to date with all variants and new applications, advances in hardware and software for data acquisition and analysis during the last years are significantly based on open software and hardware approaches. Some people are still puzzled by the apparent altruistic attitude of trying to solve others' problems, giving 
away proprietary ideas and code. Science must be reproducible and this cannot be achieved if it is not open. We strongly believe that the possibility to change the code and to tinker with the hardware, improving and adapting for a specific application is an added value for the whole community interested on mesoscopy imaging.

Acknowledgements E.J.G. acknowledges support from the Fundação para a Ciência e a Tecnologia grant SFRH/BPD/80717/2011. GGM acknowledges the support of the EMBO practical course on 3D Developmental Imaging, and of the Microscopy Unit of Faculdade de Ciências, University of Lisbon. The Ditassa burchelli flower was kindly provided by Prof. Lia Ascensão.

\section{References}

Ahrens M, Orger M, Robson D, Li J, Keller P (2013) Whole-brain functional imaging at cellular resolution using light-sheet microscopy. Nat Methods 10:413-420

Baumgart E, Kubitscheck U (2012) Scanned light sheet microscopy with confocal slit detection. Opt Express 20:21805-21814

Birk UJ, Darrell A, Konstantinides N, Sarasa-Renedo A, Ripoll J (2011) Improved reconstructions and generalized filtered back projection for optical projection tomography. Appl Opt 50(4): 392-398

Birk UJ, Rieckher M, Konstantinides N, Darrell A, Sarasa-Renedo A, Meyer H, Tavernarakis N, Ripoll J (2010) Correction for specimen movement and rotation errors for in-vivo Optical Projection Tomography. Biomed Op Express 1(1):87-96

Breuninger T, Greger K, Stelzer E (2007) Lateral modulation boosts image quality in single plane illumination fluorescence microscopy. Opt Lett 32:1938-1940

Cheddad A, Svensson C, Sharpe J, Georgsson F, Ahlgren U (2012) Image processing assisted algorithms for optical projection tomography. IEEE Trans Med Imaging 31:1-15

Cremer C, Cremer T (1978) Considerations on a laser-scanningmicroscope with high-resolution and depth of field. Microscopica Acta 81:31-44

Denk W, Strickler JH, Webb WW (1990) 2-photon laser scanning fluorescence microscopy. Science 248:73-76

Dodt H, Leischner U, Schierloh A, Jahrling N, Mauch C, Deininger K, Deussing J, Eder M, Zieglgansberger W, Becker K (2007) Ultramicroscopy: three-dimensional visualization of neuronal networks in the whole mouse brain. Nat Methods 4:331-336

Edelstein A, Amodaj N, Hoover K, Vale R, Stuurman N (2010) Computer control of microscopes using $\mu$ Manager. In: Ausubel FM et al. (eds) Current protocols in molecular biology (Chapter 14): Unit 14.20

Fahrbach F, Gurchenkov V, Alessandri K, Nassoy P, Rohrbach A (2013a) Light-sheet microscopy in thick media using scanned Bessel beams and two-photon fluorescence excitation. Opt Express 21:13824 13839

Fahrbach F, Voigt F, Schmid B, Helmchen F, Huisken J (2013b) Rapid 3D light-sheet microscopy with a tunable lens. Opt Express 21:21010 21026

Fei P, Yu Z, Wang X, Lu P, Fu Y, He Z, Xiong J, Huang Y (2012) High dynamic range optical projection tomography (HDR-OPT). Opt Express 20:8824-8836

Friedrich M, Gan Q, Ermolayev V, Harms G (2011) STED-SPIM: stimulated emission depletion improves sheet illumination microscopy eesolution. Biophys J 100:L43-L45
Gao L, Shao L, Higgins C, Poulton J, Peifer M, Davidson M, Wu X, Goldstein B, Betzig E (2012) Noninvasive imaging beyond the diffraction limit of 3D dynamics in thickly fluorescent specimens. Cell 151:1370-1385

Greger K, Swoger J, Stelzer E (2007) Basic building units and properties of a fluorescence single plane illumination microscope. Rev Sci Instrum 78:023705-023707

Gu XJ, Zhang QH, Larcom L, Jiang HB (2004) Three-dimensional bioluminescence tomography with model-based reconstruction. Opt Express 12:3996-4000

Gualda E, Vale T, Almada P, Feijo J, Martins G, Moreno N (2013) OpenSpinMicroscopy: an open-source integrated microscopy platform. Nat Methods 10:599-600

Hagerling R, Pollmann C, Andreas M, Schmidt C, Nurmi H, Adams R, Alitalo K, Andresen V, Schulte-Merker S, Kiefer F (2013) A novel multistep mechanism for initial lymphangiogenesis in mouse embryos based on ultramicroscopy. Embo J 32:629-644

Hoh J, Heinz W, Werbin J (2013) Spatial information dynamics during early zebrafish development. Dev Biol 377:126-137

Holekamp T, Turaga D, Holy T (2008) Fast three-dimensional fluorescence imaging of activity in neural populations by objective-coupled planar illumination microscopy. Neuron 57:661-672

Huisken J, Stainier D (2007) Even fluorescence excitation by multidirectional selective plane illumination microscopy (mSPIM). Opt Lett 32:2608-2610

Huisken J, Stainier D (2009) Selective plane illumination microscopy techniques in developmental biology. Development 136:19631975

Huisken J, Swoger J, Del Bene F, Wittbrodt J, Stelzer E (2004) Optical sectioning deep inside live embryos by selective plane illumination microscopy. Science 305:1007-1009

Jahrling N, Becker K, Dodt H (2009) 3D-reconstruction of blood vessels by ultramicroscopy. Org React 5:227-230

Keller P, Schmidt A, Santella A, Khairy K, Bao Z, Wittbrodt J, Stelzer E (2010) Fast, high-contrast imaging of animal development with scanned light sheet-based structured-illumination microscopy. Nat Methods 7:637-U655

Keller P, Schmidt A, Wittbrodt J, Stelzer E (2008) Reconstruction of zebrafish early embryonic development by scanned light sheet microscopy. Science 322:1065-1069

Krzic U, Gunther S, Saunders T, Streichan S, Hufnagel L (2012) Multiview light-sheet microscope for rapid in toto imaging. Nat Methods 9:730-U304

Lei M, Zumbusch A (2010) Structured light sheet fluorescence microscopy based on four beam interference. Opt Express 18:1923219241

Lindek S, Pick R, Stelzer E (1994) Confocal theta microscope with 3 objective lenses. Rev Sci Instrum 65:3367-3372

Lorbeer R, Heidrich M, Lorbeer C, Ojeda D, Bicker G, Meyer H, Heisterkamp A (2011) Highly efficient 3D fluorescence microscopy with a scanning laser optical tomograph. Opt Express 19:5419-5430

Mertz J, Kim J (2010) Scanning light-sheet microscopy in the whole mouse brain with HiLo background rejection. J Biomed Op 15: 016027

Messaoudil C, Boudier T, Sorzano C, Marco S (2007) TomoJ: tomography software for three-dimensional reconstruction in transmission electron microscopy. BMJ Bioinforma 8:84

Ntziachristos V (2010) Going deeper than microscopy: the optical imaging frontier in biology. Nat Methods 7:603-614

Olarte O, Licea-Rodriguez J, Palero J, Gualda E, Artigas D, Mayer J, Swoger J, Sharpe J, Rocha-Mendoza I, Rangel-Rojo R, LozaAlvarez P (2012) Image formation by linear and nonlinear digital scanned light-sheet fluorescence microscopy with Gaussian and Bessel beam profiles. Biomed Op Express 3:1492-1505

Panier T, Romano SA, Olive R, Pietri T, Sumbre G, Candelier R, Debregeas G (2013) Fast functional imaging of multiple brain 
regions in intact zebrafish larvae using Selective Plane Illumination Microscopy. Frontiers Neural Circuits 7:67

Pitrone P, Schindelin J, Stuyvenberg L, Preibisch S, Weber M, Eliceiri K, Huisken J, Tomancak P (2013) OpenSPIM: an open-access lightsheet microscopy platform. Nat Methods 10:598-599

Planchon T, Gao L, Milkie D, Davidson M, Galbraith J, Galbraith C, Betzig E (2011) Rapid three-dimensional isotropic imaging of living cells using Bessel beam plane illumination. Nat Methods 8:417-U468

Preibisch S, Rohlfing T, Hasak MP, Tomancak P (2008) Mosaicing of single plane illumination microscopy images using groupwise registration and fast content-based image fusion — art. no. 69140E. In Medical Imaging 2008 Conference, Vol. 6914, pp E9140-E9140. San Diego, CA: Med SAAP, Soc AP, Radiol CA, Surg, Soc Imaging S, Technol, Soc MIP, Radiol Soc N, Amer, Med SII, Soc Mole Imaging DSC

Preibisch S, Saalfeld S, Schindelin J, Tomancak P (2010) Software for bead-based registration of selective plane illumination microscopy data. Nat Methods 7:418-419

Ritter J, Veith R, Veenendaal A, Siebrasse J, Kubitscheck U (2010) Light sheet microscopy for single molecule tracking in living tissue. Plos One 5:e11639

Rubio-Guivernau JL, Gurchenkov V, Luengo-Oroz MA, Duloquin L, Bourgine P, Santos A, Peyrieras N, Ledesma-Carbayo MJ (2012) Wavelet-based image fusion in multi-view three-dimensional microscopy. Bioinformatics 28:238-245

Schindelin J, Arganda-Carreras I, Frise E, Kaynig V, Longair M, Pietzsch T, Preibisch S, Rueden C, Saalfeld S, Schmid B, Tinevez J, White D, Hartenstein V, Eliceiri K, Tomancak P, Cardona A (2012) Fiji: an opensource platform for biological-image analysis. Nat Methods 9:676682

Schmid B, Shah G, Scherf N, Weber M, Thierbach K, Campos C, Roeder I, Aanstad P, Huisken J (2013) High-speed panoramic light-sheet microscopy reveals global endodermal cell dynamics. Nat Commun 4

Sharpe J, Ahlgren U, Perry P, Hill B, Ross A, Hecksher-Sorensen J, Baldock R, Davidson D (2002) Optical projection tomography as a tool for 3D microscopy and gene expression studies. Science 296: $541-545$

Shaw PJ, Agard DA, Hiraoka Y, Sedat JW (1989) TIlted view reconstruction in optical microscopy - 3-dimensional reconstruction of Drosophila melanogaster embryo nuclei. Biophys J 55:101-110
Silvestri L, Bria A, Sacconi L, Iannello G, Pavone F (2012) Confocal light sheet microscopy: micron-scale neuroanatomy of the entire mouse brain. Opt Express 20:20582-20598

Swoger J, Muzzopappa M, Lopez-Schier H, Sharpe J (2011) 4D retrospective lineage tracing using SPIM for zebrafish organogenesis studies. J Biophotonics 4:122-134

Swoger J, Verveer P, Greger K, Huisken J, Stelzer E (2007) Multi-view image fusion improves resolution in three-dimensional microscopy. Opt Express 15:8029-8042

Tomer R, Khairy K, Amat F, Keller P (2012) Quantitative high-speed imaging of entire developing embryos with simultaneous multiview light-sheet microscopy. Nat Methods 9:755-U181

Truong T, Supatto W, Koos D, Choi J, Fraser S (2011) Deep and fast live imaging with two-photon scanned light-sheet microscopy. Nat Methods 8:757-U102

Vinegoni C, Razansky D, Figueiredo J, Nahrendorf M, Ntziachristos V, Weissleder R (2009) Normalized Born ratio for fluorescence optical projection tomography. Opt Lett 34:319-321

Voie A, Burns D, Spelman F (1993) Orthogonal-plane fluorescence optical sectioning - 3-dimensional imaging of macroscopic biological specimens. J Microsc-Oxford 170:229-236

Walls J, Sled J, Sharpe J, Henkelman R (2007) Resolution improvement in emission optical projection tomography. Phys Med Biol 52:27752790

Wang G, Li Y, Jiang M (2004) Uniqueness theorems in bioluminescence tomography. Med Phys 31:2289-2299

Weber M, Huisken J (2012) Omnidirectional microscopy. Nat Methods 9: 656-657

Wohland T, Shi X, Sankaran J, Stelzer E (2010) Single Plane Illumination Fluorescence Correlation Spectroscopy (SPIM-FCS) probes inhomogeneous three-dimensional environments. Opt Express 18: 10627-10641

Wu Y, Ghitani A, Christensen R, Santella A, Du Z, Rondeau G, Bao Z, Colon-Ramos D, Shroff H (2011) Inverted selective plane illumination microscopy (iSPIM) enables coupled cell identity lineaging and neurodevelopmental imaging in Caenorhabditis elegans. Proc Natl Acad Sci U S A 108:17708-17713

Zanacchi F, Lavagnino Z, Donnorso M, Del Bue A, Furia L, Faretta M, Diaspro A (2011) Live-cell 3D super-resolution imaging in thick biological samples. Nat Methods 8:1047-1049 